\title{
COPD is associated with an increased risk of peripheral artery disease and mortality
}

\author{
Natalie Terzikhan ${ }^{1,2}$, Lies Lahousse ${ }^{2,3}$, Katia M.C. Verhamme ${ }^{4}$, \\ Oscar H. Franco ${ }^{2}$, M. Arfan Ikram², Bruno H. Stricker ${ }^{2,5}$ and Guy G. Brusselle ${ }^{1,2,6}$
}

Affiliations: ${ }^{1}$ Dept of Respiratory Medicine, Ghent University Hospital, Ghent, Belgium. ${ }^{2}$ Dept of Epidemiology, Erasmus MC - University Medical Center Rotterdam, Rotterdam, The Netherlands. ${ }^{3}$ Dept of Bioanalysis, Faculty of Pharmaceutical Sciences, Ghent University, Ghent, Belgium. ${ }^{4}$ Dept of Medical Informatics, Erasmus MC - University Medical Center Rotterdam, Rotterdam, The Netherlands. ${ }^{5}$ Dept of Internal Medicine, Erasmus MC - University Medical Center Rotterdam, Rotterdam, The Netherlands. ${ }^{6}$ Dept of Respiratory Medicine, Erasmus MC - University Medical Center Rotterdam, Rotterdam, The Netherlands.

Correspondence: Bruno H. Stricker, Dept of Epidemiology, Erasmus MC - University Medical Center Rotterdam, PO Box 2040, 3000 CA Rotterdam, The Netherlands. E-mail: b.strickerderasmusmc.nl

ABSTRACT Patients with chronic obstructive pulmonary disease (COPD) commonly present with multimorbidity. We aimed to investigate the association between COPD and the development of peripheral arterial disease (PAD) in the general population, and how this might affect mortality among individuals with COPD.

We included 3123 participants of the population-based Rotterdam Study without PAD at baseline (mean age 65 years; $57.4 \%$ female). The association between COPD at baseline and PAD during follow-up was studied using logistic regression (PAD being indicated by an ankle-brachial index (ABI) of 0.9 or less). Cox regression was used for mortality analysis and interaction terms were used to investigate mortality risk modification by PAD.

The presence of COPD was associated with incident PAD (adjusted odds ratio 1.9, 95\% CI 1.1-3.2). Mortality rates per 100000 person-years were as follows: 10.0 in individuals without COPD or PAD, 18.4 in those with COPD only, 16.1 in those with PAD only and 30.1 in individuals with both COPD and PAD. No statistical interaction was found between PAD and COPD on risk of dying.

Individuals with COPD have an almost doubled risk of developing PAD. Although PAD does not modify the association between COPD and mortality, people suffering from both diseases have substantially higher mortality rates.

@ERSpublications

Individuals with COPD have an almost doubled risk of developing peripheral arterial disease (PAD). People suffering from both COPD and PAD have substantially higher mortality rates. http://ow.ly/IyOx30m0IBo

Cite this article as: Terzikhan N, Lahousse L, Verhamme KMC, et al. COPD is associated with an increased risk of peripheral artery disease and mortality. ERJ Open Res 2018; 4: 00086-2018 [https:// doi.org/10.1183/23120541.00086-2018].

Received: June 122018 | Accepted after revision: Sept 232018

Clinical trials: The Rotterdam Study has been entered into the Netherlands National Trial Register (NTR; www. trialregister.nl) and into the WHO International Clinical Trials Registry Platform (ICTRP; www.who.int/ictrp/network/ primary/en/) under shared catalogue number NTR6831.

Copyright $\odot$ ERS 2018. This article is open access and distributed under the terms of the Creative Commons Attribution Non-Commercial Licence 4.0. 


\section{Introduction}

Chronic obstructive pulmonary disease (COPD) is the third major cause of death worldwide [1]. Beyond airflow limitation and respiratory impairment, COPD patients often suffer from multimorbidities, the most prominent of which are vascular diseases $[2,3]$.

Atherosclerosis is known as a major cause of symptomatic vascular disease. One of the manifestations of atherosclerosis is peripheral arterial disease (PAD), which refers to the occlusion of the arteries in the lower limbs. PAD is often asymptomatic but can be present in advanced stages in the form of intermittent claudication, leg pain at rest and, rarely, non-healing wounds, ulcerations and gangrene [4]. Patients with PAD have comparable but not identical risk factor profiles to patients with coronary heart disease, with systemic inflammation, diabetes and smoking being the predominant risks in PAD [5-8].

The prevalence of PAD in individuals with COPD shows a wide range of variation depending on the severity of COPD in the study population. Its prevalence was found to be $8 \%$ in Asian COPD patients, $8.8 \%$ in the German COSYCONET study of COPD patients followed in secondary care and $36.8 \%$ in COPD patients hospitalised for a severe exacerbation [9-11].

A previous study by PECCI et al. [10] demonstrated that PAD was asymptomatic in a large proportion of COPD patients and was associated with more severe lung disease than in COPD subjects without PAD. Therefore, longitudinal studies investigating the association between COPD and incident PAD are needed [12]. In addition, although $\mathrm{PAD}$ is known to have a significant impact on mortality, it is unknown whether the risk of mortality is higher in patients with both COPD and PAD. Therefore, the objectives of our study were as follows: 1) to investigate the association between COPD and incident PAD in a longitudinal cohort, and 2) to elucidate the effect of PAD on mortality in subjects with COPD in a community-dwelling population of middle-aged and older subjects.

\section{Methods}

Setting

The present study was embedded within the Rotterdam Study, an ongoing prospective population-based cohort which was enroled to investigate the occurrence of, as well as risk factors for, chronic diseases in the general population. The aims and methods of the Rotterdam Study have previously been published in detail [13]. In short, the Rotterdam Study started in 1990, with inhabitants of the Ommoord district of Rotterdam being invited to the research centre for follow-up examination every 3-4 years. Participants were additionally monitored continuously for morbidity and mortality through linkage of both general practitioner (GP) and municipality records to the study. The present study was conducted using data from the first cohort of the Rotterdam Study (RS-I) and comprises two parts. The first longitudinal analysis was performed in order to study the association between COPD (measured at baseline, between 1990 and 1993) and newly diagnosed PAD (measured between 1996 and 2000) (figure 1). The second analysis aimed to investigate the effect of PAD on mortality in subjects with COPD (measured between 1996 and 2016). The Rotterdam Study has been approved by the Medical Ethics Committee of the Erasmus MC (registration number MEC 02.1015) and by the Dutch Ministry of Health, Welfare and Sport (Population Screening Act WBO, license number 1071272-159521-PG). All participants provided written informed consent to participate in the study and to have their information obtained from treating physicians.

\section{COPD diagnosis}

We validated whether participants had COPD by reviewing the medical charts, including outpatient clinic reports and hospital discharge letters, of all those who used medication for obstructive lung disease for at least 6 months (anatomical therapeutic chemical (ATC) classification code R03) or who stated they had COPD in a questionnaire. COPD cases were defined as having a medical COPD diagnosis supported by clinical presentation and/or obstructive lung function [14]. The index date was defined as the date of diagnosis of COPD as described in the medical charts or the date of the first prescription for COPD medication in patients with COPD, whichever came first. In this study COPD data was used at baseline for the first objective and at follow-up for the second objective.

\section{Assessment of PAD}

The method of measuring the ankle-brachial index (ABI) has been described previously [15]. In short, blood pressure (BP) in the arm (systolic BP) was determined by calculating the mean of two successive measurements at the right brachial artery while the participant was in a sitting position (using a random-zero sphygmomanometer) [15]. In addition, the systolic BP of the posterior tibial artery was measured in both the left leg and the right leg while the participant was in a supine position (using a random-zero sphygmomanometer and an $8-\mathrm{MHz}$ continuous-wave Doppler probe (Huntleigh $500 \mathrm{D}$, Huntleigh Technology)). The ABI was defined as the ratio of systolic BP at the ankle to systolic BP at the 


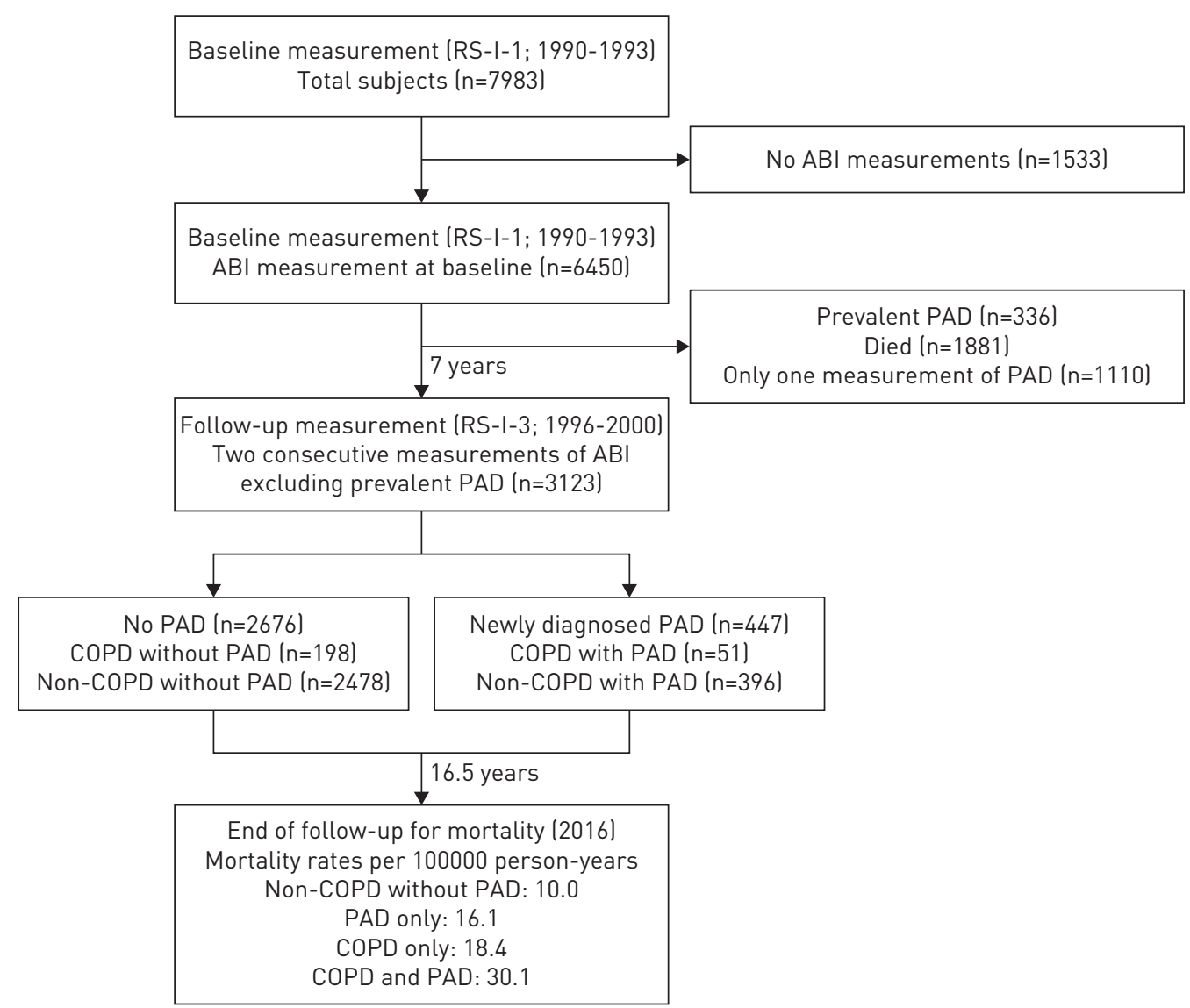

FIGURE 1 Flow chart to investigate the association between chronic obstructive pulmonary disease (COPD) and newly diagnosed peripheral arterial disease (PAD). ABI: ankle-brachial index; RS-I-1: the first visit of the first cohort of the Rotterdam Study; RS-I-3: the third visit of the first cohort of the Rotterdam Study.

arm and was calculated for each leg. The presence of PAD was defined as an ABI of 0.90 or less in at least one leg [5]. Patients with prevalent PAD at baseline were excluded and only newly diagnosed (incident) PAD during follow-up was considered as an outcome. Severe PAD was defined as an ABI of less than 0.6 [11]. Besides the ABI measurements, we used the criteria of the World Health Organization (WHO)/Rose questionnaire, which was incorporated in the home interview [16], to investigate intermittent claudication and capture symptomatic PAD.

\section{Follow-up for mortality}

To investigate whether PAD increases the risk of all-cause mortality in subjects with or without COPD, 3123 individuals with two ABI measurements were followed from the second PAD measurement until mortality, or until the last visit to the study centre.

Information on mortality was obtained from the municipality of Rotterdam and was additionally validated with data from study participants medical records, as kept by their GPs and as described in detail previously [17]. Mortality data was complete until August 2016.

\section{Covariables}

Relevant covariables at baseline were obtained for each analysis using interview, blood sampling and physical examination [18]. Smoking status was assessed by interview and categorised as never, former or current smoker, or as ever or never smoker. Body mass index (BMI) was calculated by dividing body weight (in $\mathrm{kg}$ ) by height (in $\mathrm{m}^{2}$ ). Hypertension was defined as a systolic BP $\geqslant 140 \mathrm{mmHg}$, a diastolic BP $\geqslant 90 \mathrm{mmHg}$, or the use of BP-lowering drugs. Subjects were classified as statin users if they had received at least one prescription for statins at baseline. Information on statin use was obtained from interview and from pharmacies. Type-2 diabetes mellitus was defined, in accordance with recent WHO guidelines, by fasting blood glucose $\geqslant 7.0 \mathrm{mmol} \cdot \mathrm{L}^{-1}$, non-fasting blood glucose $\geqslant 11.1 \mathrm{mmol} \cdot \mathrm{L}^{-1}$ (in the absence of fasting samples), or the use of blood glucose lowering medication [17]. Concentrations of high-density lipoprotein 
(HDL) cholesterol and total cholesterol were measured from blood samples using an automated enzymatic method [19]. The HDL cholesterol/total cholesterol ratio was calculated by dividing HDL blood concentrations by total cholesterol concentrations. Ethnicity was based on genetic ancestry data or interview data in the absence of genetic information. High-sensitivity C-reactive protein (hs-CRP) was measured using rate near infrared particle immunoassay (Immage Immunochemistry System, Beckman Coulter, Fullerton, CA, USA).

\section{Statistical analysis}

To study population characteristics the Chi-squared test and the independent sample t-test were used to test differences between individuals with or without COPD. We determined the association between baseline COPD and the development of PAD (assessed during a follow-up visit), using logistic regression after exclusion of individuals with prevalent PAD at baseline. For the association between COPD and newly diagnosed PAD, we adjusted for covariables that were considered risk factors for atherosclerosis and cardiovascular disease. The following potential confounders were considered: age, sex, smoking status, smoking duration in pack-years, BMI, hypertension, hs-CRP, statin use, HDL/cholesterol ratio, ethnicity and diabetes mellitus. Missing data on covariables were imputed using the expectation maximization method and effect modification was tested with interaction terms. Additional sensitivity analyses were performed by adjusting for hs-CRP (as a marker for inflammation) and by excluding individuals with incident COPD or individuals with an ABI greater than 1.4 (as a marker for arterial stiffening [15]) from the analysis.

As a secondary objective, we aimed to study the impact of PAD on the risk of mortality among individuals with COPD. For this analysis four groups were defined as follows (all assessed at the second PAD round): 1) individuals without COPD and without PAD, 2) individuals with COPD only, 3) individuals with PAD only, and 4) individuals with both COPD and PAD. Mortality rates were obtained by dividing the number of deaths by the total number of person-years of subjects at risk and were presented per 100000 person-years. Confidence intervals around these rates were calculated using a Poisson distribution. Follow-up time for all subjects was defined as the time period between the start of the second analysis (where incident PAD was measured) and death or the last visit to the study centre. Sensitivity analysis was performed by censoring for incident COPD during follow-up. Median follow-up time was estimated using the reverse Kaplan-Meier method. Crude survival curves were obtained using the Kaplan-Meier survival method. The Cox proportional hazard model was used to calculate mortality hazard ratios and was adjusted for age, sex, smoking duration in pack-years, hypertension, HDL/cholesterol ratio and diabetes

\section{TABLE 1 Baseline characteristics of all subjects without peripheral arterial disease at baseline, stratified by chronic obstructive pulmonary disease (COPD) status}

\begin{tabular}{lcccr} 
Characteristic & Overall & No COPD & COPD & p-value $^{\#}$ \\
\hline Subjects & $3123(100)$ & $3038(97)$ & $85(3)$ & \\
Age years & $65.14 \pm 6.6$ & $65.12 \pm 6.6$ & $65.69 \pm 5.6$ & 0.43 \\
Female & $1785(57.2)$ & $1745(57.4)$ & $40(47.1)$ & 0.07 \\
BMI kg.m -2 $_{\text {Smoking status }}$ & $26.27 \pm 3.5$ & $26.28 \pm 3.5$ & $26.17 \pm 3.1$ & 0.78 \\
$\quad$ Never & $1069(35.4)$ & $1054(35.8)$ & $15(18.5)$ & $<0.01$ \\
$\quad$ Former & $1394(46.1)$ & $1348(45.8)$ & $46(56.8)$ & \\
$\quad$ Current & $560(18.5)$ & $540(18.4)$ & $20(24.7)$ & \\
Ever smoking & $1954(64.6)$ & $1888(64.2)$ & $66(81.5)$ & $<0.01$ \\
Smoking duration pack-years" & $25.5 \pm 21.3$ & $25.3 \pm 21.2$ & $33.5 \pm 24.1$ & $<0.01$ \\
Diabetes mellitus & $380(12.6)$ & $367(12.5)$ & $13(15.9)$ & 0.47 \\
HDL/cholesterol ratio & $0.21 \pm 0.1$ & $0.21 \pm 0.1$ & $0.22 \pm 0.1$ & 0.13 \\
Cholesterol lowering drugs & $72(2.3)$ & $70(2.3)$ & $2(2.4)$ & 0.98 \\
Hypertension & $1481(47.4)$ & $1446(47.6)$ & $35(41.2)$ & 0.29 \\
BP lowering drugs & $770(24.7)$ & $743(24.5)$ & $27(31.8)$ & 0.12 \\
hs-CRP & $2.44 \pm 3.3$ & $2.42 \pm 3.3$ & $3.02 \pm 3.0$ & 0.11 \\
Inhaled therapy & $124(4.0)$ & $64(2.1)$ & $60(70.6)$ & $<0.01$ \\
\end{tabular}

Data are presented as $\mathrm{n}(\%)$ or mean $\pm \mathrm{SD}$. Data are original data without imputed values. In the total population, missing values were present for smoking (3.2\%), smoking duration in pack-years (7.1\%), BMI $(0.2 \%)$ and diabetes mellitus (3.5\%). BMI: body mass index; HDL: high-density lipoprotein; BP: blood pressure; hs-CRP: high-sensitivity C-reactive protein. ${ }^{\#}$ : $p$-values represent the difference between COPD groups; " : smoking duration in pack-years is presented for former and current smokers only. 
mellitus. We used SPSS version 21 (IBM Corp, Armonk, NY, USA) and R-software (The R Project for Statistical computing; www.r-project.org) for all analyses.

\section{Results}

The characteristics of the population for follow-up $(n=3123)$ are presented in table 1 . The mean age \pm SD was $65.1 \pm 6.6$ years and $57.2 \%$ of subjects were women. The prevalence of ever smokers was $65 \%$ in the total population and $67.1 \%$ in individuals with incident PAD (table 1 and table 2). Considering individuals with PAD, almost all men (89.1\%) and $48.3 \%$ of women were ever smokers. In total, 56 out of 3123 individuals $(1.8 \%)$ had severe PAD based on an ABI of less than 0.6. Only 12 out of 56 subjects with severe PAD (21.4\%) indicated intermittent claudication on their questionnaire.

\section{COPD and the association with newly diagnosed PAD}

During a median follow-up of 7.4 years, 447 patients (12.9\%) developed incident PAD. A statistically significant association was found between COPD and newly diagnosed PAD during follow-up. The ageand sex-adjusted odds ratio (OR) was 1.89 (95\% CI 1.13-3.17) and 1.87 (95\% CI 1.10-3.18) after additional adjustment for smoking duration in pack-years, hypertension, HDL/cholesterol ratio and diabetes mellitus (table 2). This association remained significant upon additional adjustment for baseline hs-CRP levels (adjusted OR 1.86, 95\% CI 1.10-3.16), exclusion of individuals ( $\mathrm{n}=186)$ who developed COPD during follow-up (adjusted OR 1.93, 95\% CI 1.13-3.29) and upon exclusion of individuals ( $\mathrm{n}=495)$ with an ABI of greater than 1.40 (adjusted OR 1.95, 95\% CI 1.13-3.36). The association between COPD and incident PAD was stronger in males (adjusted OR 2.59, 95\% CI 1.04-5.16) than females (adjusted OR $1.22,95 \%$ CI $0.53-2.87$ ). However, the test for interaction between COPD and sex was not significant ( $\mathrm{p}=0.18$ for interaction). Stratified analysis by smoking status revealed that the overall association between COPD and newly developed PAD was driven by the ever smoker group ( $\mathrm{p}=0.41$ for interaction) (table 2).

\section{Disease status and risk of all-cause mortality}

We investigated the impact of PAD on mortality by following all subjects from the time when the second PAD measurement was performed $(n=3123)$. The general characteristics of the population which was followed-up for mortality are presented in table 3 . The prevalence of PAD in COPD cases was $20.5 \%$ (51 out of 249 individuals) while the prevalence of PAD in non-COPD cases was $13.8 \%$ (396 out of 2874

\begin{tabular}{|c|c|c|c|c|c|c|}
\hline \multirow[t]{2}{*}{ Stratification } & \multicolumn{3}{|c|}{ Subjects } & \multirow{2}{*}{$\begin{array}{c}\text { Model } 1 \# \\
\text { OR }(95 \% \text { CI) }\end{array}$} & \multirow{2}{*}{$\begin{array}{c}\text { Model } 2^{\pi} \\
\text { OR }(95 \% \text { CI) }\end{array}$} & \multirow[t]{2}{*}{ p-value ${ }^{+}$} \\
\hline & Overall & No PAD & PAD & & & \\
\hline Overall & $3123(100)$ & $2676(100)$ & $447(100)$ & & & \\
\hline Non-COPD & 3038 (97.3) & $2611(97.6)$ & 427 (95.5) & Ref & & \\
\hline COPD & 85 (2.7) & $65(2.4)$ & $20(5.0)$ & $1.89(1.13-3.18)$ & $1.87(1.10-3.18)$ & \\
\hline Sex & & & & & & 0.18 \\
\hline \multicolumn{7}{|l|}{ Male } \\
\hline Non-COPD & $1293(41.4)$ & 1122 (41.9) & $171(38.3)$ & Ref & & \\
\hline COPD & 45 (1.4) & 32 (1.2) & 13 (2.9) & $2.61(1.33-5.12)$ & $2.59(1.04-5.16)$ & \\
\hline \multicolumn{7}{|l|}{ Female } \\
\hline Non-COPD & 1745 (55.9) & $1489(55.6)$ & $256(57.3)$ & Ref & & \\
\hline COPD & 40 (1.3) & $33(1.2)$ & $7(1.6)$ & $1.24(0.54-2.87)$ & $1.22(0.53-2.87)$ & \\
\hline Smoking status & & & & & & 0.41 \\
\hline \multicolumn{7}{|l|}{ Never smoker } \\
\hline Non-COPD & 1066 (34.1) & $921(34.4)$ & $145(32.4)$ & Ref & & \\
\hline COPD & $15(0.5)$ & $13(0.5)$ & $2(0.4)$ & $1.00(0.21-4.47)$ & $1.20(0.26-5.57)$ & \\
\hline \multicolumn{7}{|l|}{ Ever smoker } \\
\hline Non-COPD & $1972(63.1)$ & $1690(63.2)$ & $282(63.1)$ & Ref & & \\
\hline COPD & 70 (2.2) & 52 (1.9) & $18(4.0)$ & $2.00(1.14-3.50)$ & $1.98(1.12-3.49)$ & \\
\hline $\begin{array}{l}\text { Data are present } \\
\text { in bold are statis } \\
\text { sex; ๆ: model } 2 \\
\text { lipoprotein/chole } \\
\text { interaction term }\end{array}$ & $\begin{array}{l}\text { as } n(\%) \text { or } \\
\text { Ily significa } \\
\text { adjusted } f \\
\text { rol ratio ar } \\
\text { nodel } 2 \text {. }\end{array}$ & $\begin{array}{l}\text { atio } 195 \% \text { C } \\
\text { Oge, sex, } \\
\text { diabetes }\end{array}$ & $\begin{array}{l}\text { Data for } \\
\text { tio; Ref: } r \\
\text { noking du } \\
\text { llitus; }{ }^{+} \text {: }\end{array}$ & $\begin{array}{l}\text { oking is represe } \\
\text { rence value. }{ }^{\#}: \\
\text { tion in pack-yea } \\
\text { alue for intera }\end{array}$ & $\begin{array}{l}\text { ed by imputed va } \\
\text { del } 1 \text { is adjusted } \\
\text {, hypertension, } \\
\text { on, calculated by }\end{array}$ & $\begin{array}{l}\text { es. Values } \\
\text { or age and } \\
\text { gh-density } \\
\text { adding an }\end{array}$ \\
\hline
\end{tabular}




\section{TABLE 3 Baseline characteristics of all subjects followed for mortality at the second} peripheral arterial disease (PAD) assessment

\begin{tabular}{|c|c|c|c|c|c|}
\hline & Overall & No COPD or PAD & COPD only \# & PAD only & COPD and $P A D^{\#}$ \\
\hline Subjects & 3123 & 2478 & 198 & 396 & 51 \\
\hline Age years & $71.73 \pm 6.58$ & $71.13 \pm 6.36$ & $72.79 \pm 5.95$ & $74.58 \pm 7.21$ & $74.84 \pm 6.89$ \\
\hline Women & $1785(57.20)$ & 1435 (57.90) & $87(43.90)$ & $246(62.10)$ & $17(33.30)$ \\
\hline $\mathrm{BMI} \mathrm{kg} \cdot \mathrm{m}^{-2}$ & $26.87 \pm 3.89$ & $26.94 \pm 3.86$ & $26.91 \pm 3.78$ & $26.55 \pm 4.08$ & $25.83 \pm 3.72$ \\
\hline Ever smoker & $2049(65.60)$ & $1580(63.80)$ & $163(82.30)$ & $261(65.9)$ & 45 (88.2) \\
\hline Smoking duration pack-years & $25.47 \pm 22.50$ & $23.99 \pm 22.01$ & $34.13 \pm 26.62$ & $26.49 \pm 19.85$ & $39.78 \pm 25.63$ \\
\hline Diabetes mellitus & $816(26.10)$ & $608(24.50)$ & $63(31.80)$ & $130(32.80)$ & $15(29.40)$ \\
\hline HDL/cholesterol ratio & $0.24 \pm 0.07$ & $0.25 \pm 0.07$ & $0.25 \pm 0.08$ & $0.23 \pm 0.06$ & $0.25 \pm 0.08$ \\
\hline
\end{tabular}

Data are presented as $\mathrm{n}(\%)$ or mean $\pm \mathrm{SD}$, and are represented by imputed values. Data are stratified as subjects without chronic obstructive pulmonary disease (COPD) and without PAD, subjects with COPD only, subjects with PAD only and subjects with both COPD and PAD. BMI: body mass index; HDL: high-density lipoprotein. \#: the total number of individuals with COPD at follow-up was 249 (COPD only (n=198) plus COPD and PAD ( $n=51)$ ). This represents those subjects with COPD at baseline ( $n=85$; table 1$)$ plus those individuals that acquired COPD during follow-up. ": smoking duration in pack-years is presented for former and current smokers only.

individuals). We stratified the study population into the following four groups: 1) no COPD or PAD $(\mathrm{n}=2478), 2)$ COPD only $(\mathrm{n}=198), 3)$ PAD only $(\mathrm{n}=396)$, and 4) comorbid COPD and PAD $(\mathrm{n}=51)$. Median follow-up time for mortality was 16.5 years. At the end of follow-up, 1805 individuals (58\%) had died. The mortality rate in the group without COPD and without PAD was 10.0 (95\% CI 9.47-10.62) per 100000 person-years. Subjects with COPD only and subjects with PAD only had comparable mortality rates of 18.4 (95\% CI 15.49-21.58) and 16.1 (95\% CI 14.17-18.16) per 100000 person-years, respectively. In subjects with both COPD and PAD, the mortality rate was the highest at 30.1 (95\% CI 22.01-40.11) per 100000 person-years. The Kaplan-Meier survival curves in the different groups are represented in figure 2. Age- and sex-adjusted hazard ratios for mortality, as compared to individuals without COPD and without PAD, were 1.41 (95\% CI 1.24-1.61) for subjects with PAD only, 1.50 (95\% CI 1.26-1.77) for subjects with COPD only and 2.23 (95\% CI 1.66-3.00) for subjects with both COPD and PAD. After additional adjustment for smoking duration in pack-years, hypertension, $\mathrm{HDL} / \mathrm{cholesterol} \mathrm{ratio} \mathrm{and} \mathrm{diabetes} \mathrm{mellitus}$ the hazard ratios for mortality were 1.36 (95\% CI 1.19-1.54) for subjects with PAD only, 1.52 (95\% CI 1.28-1.80) for subjects with COPD only and 2.30 (95\% CI 1.71-3.09) for subjects with both COPD and PAD. We did not find effect modification by PAD on the multiplicative scale ( $\mathrm{p}=0.82$ for interaction). In addition, sensitivity analysis after censoring for incident COPD during follow-up did not materially change the results.

\section{Discussion}

To our knowledge, this is the first longitudinal population-based study that has investigated the association between COPD and incident PAD, and the influence of PAD on mortality rates in individuals with COPD. We observed that individuals with COPD have a higher risk of developing PAD and that the risk of mortality was highest in people with both diseases.

Smoking is a well-known risk factor for both COPD and PAD. However, in our study, the association between COPD and PAD remained statistically significant upon adjustment for cumulative smoking

FIGURE 2 Kaplan-Meier curves of mortality in different groups according to the presence or absence of chronic obstructive pulmonary disease (COPD) and peripheral arterial disease (PAD).

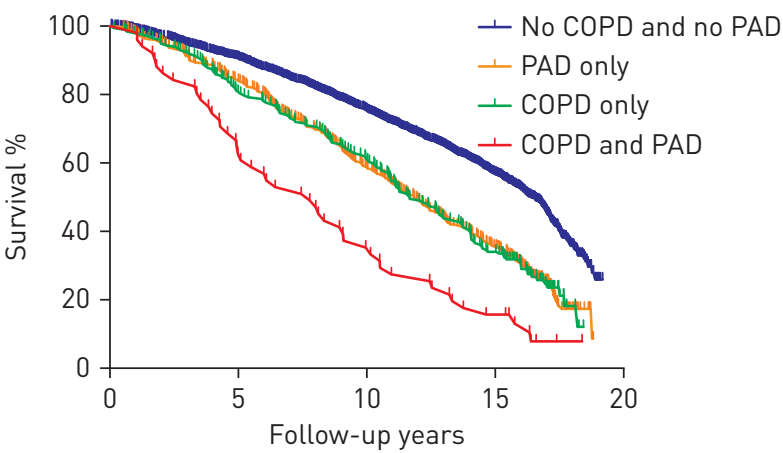


history (in pack-years). Additionally, increased C-reactive protein (CRP) levels are known to be an important risk factor for PAD and CRP is also elevated in a subgroup of patients with COPD [20]. However, adjusting for baseline hs-CRP levels did not change the overall association substantially. Despite the potential for residual confounding or other unmeasured mediators of systemic inflammation, such as tumour necrosis factor- $\alpha$ (TNF- $\alpha$ ), interleukin- $1 \beta$ (IL-1 $\beta$ ) or matrix metalloproteinases, we hypothesise that COPD-related mechanisms beyond smoking and systemic inflammation might contribute to the onset of PAD.

COPD and PAD have a well-known impact on mortality, however, no study in the literature has investigated the impact of PAD on the association between COPD and all-cause mortality. We observed a higher risk of mortality in individuals when COPD and PAD co-occur, although there was no formal statistical interaction between both diseases on a multiplicative scale. The observed mortality rates in individuals with PAD or with COPD are in line with literature reports. The European Respiratory Society (ERS) white book (www.erswhitebook.org) reported an age-standardised mortality rate for COPD of approximately 18 per 100000 inhabitants per year (to the European Standard Population), while unstandardized all-cause mortality in the subgroup of individuals with COPD only was 18.4 per 100000 person-years. In addition, in a group of patients with asymptomatic PAD, DieHM et al. [21] reported a mortality rate ratio of 1.4 after adjustment for known cardiovascular risk factors. We observed a similar adjusted hazard ratio of 1.4 in our population, which highlights the robustness of our data.

Our findings have important implications for disease management [22]. As COPD is associated with PAD development, with PAD often being asymptomatic, patients with COPD might benefit from routine ABI screening for the timely diagnosis of PAD. Apart from its cost-effectiveness, early targeted screening and treatment of asymptomatic PAD is likely to improve health by preventing future cardiovascular disease [23].

The strengths of this study are the prospective longitudinal design and the standardised data collection. However, our study also has some limitations. First, information on PAD was only gathered at the study centre, during study visits, implying that actual dates of PAD onset were missing and preventing us from conducting a time-to-PAD development analysis. Secondly, since spirometry measurements were not routinely performed in the early rounds of the Rotterdam Study, the incident date for COPD was based solely on a clinical diagnosis of COPD and not all clinically diagnosed COPD subjects were still alive to be confirmed by spirometry at the research centre at a later date. Thirdly, although we have evaluated all available known potential confounders of the association between COPD and PAD development, residual confounding might still explain part of this association. Finally, including patients with two ABI measurements at two subsequent visits might lead to a selection towards healthier individuals.

\section{Conclusion}

Subjects with COPD have a higher risk of developing PAD. People with both COPD and PAD have a substantially increased risk of death. Consequently, early detection of PAD and preventive actions in people with COPD should receive more attention in clinical respiratory care.

Acknowledgements: The authors are grateful to the study participants, the staff from the Rotterdam Study, and the participating GPs and pharmacists.

Conflict of interest: N. Terzikhan has nothing to disclose. L. Lahousse reports personal fees (for expert consultation) from Boehringer Ingelheim GmbH and Novartis, and grants (for unrestricted research) from AstraZeneca and Chiesi, outside the submitted work. K.M.M. Verhamme has received grants from GSK and Novartis, and works for a research group which has received unconditional research grants from Pfizer/Boehringer Ingelheim, Novartis, GSK and Yamanouchi. None of which are related to the content of this work. O.H. Franco reports grants from Nestle (for child nutrition) and Metagenics (for womens health), outside the submitted work. A. Ikram has nothing to disclose. B.H. Stricker has nothing to disclose. G.G. Brusselle has nothing to disclose.

Support statement: This work was supported by a grant from the Fund for Scientific Research Flanders (FWO; project G035014N). The Rotterdam Study is funded by Erasmus MC and Erasmus University Rotterdam; the Netherlands Organisation for Health Research and Development (ZonMw); the Research Institute for Diseases in the Elderly (RIDE); the Ministry of Education, Culture and Science; the Ministry for Health, Welfare and Sports; the European Commission (DG XII); and the Municipality of Rotterdam. Funding information for this article has been deposited with the Crossref Funder Registry.

\section{References}

1 Lozano R, Naghavi M, Foreman K, et al. Global and regional mortality from 235 causes of death for 20 age groups in 1990 and 2010: a systematic analysis for the Global Burden of Disease Study 2010. Lancet 2012; 380: 2095-2128.

2 Barnes PJ. Senescence in COPD and its comorbidities. Annu Rev Physiol 2017; 79: 517-539.

3 Negewo NA, Gibson PG, McDonald VM. COPD and its comorbidities: impact, measurement and mechanisms. Respirology 2015; 20: 1160-1171. 
Patel MR, Conte MS, Cutlip DE, et al. Evaluation and treatment of patients with lower extremity peripheral artery disease: consensus definitions from Peripheral Academic Research Consortium (PARC). J Am Coll Cardiol 2015; 65: 931-941.

5 Halliday A, Bax JJ. The 2017 ESC guidelines on the diagnosis and treatment of peripheral arterial diseases, in collaboration with the European Society for Vascular Surgery (ESVS). Eur J Vasc Endovasc Surg 2018; 55: 301-302.

6 Bah F, Bhimji SS. Peripheral Arterial Disease. Treasure Island, StatPearls Publishing, 2018.

7 Hirsch AT, Criqui MH, Treat-Jacobson D, et al. Peripheral arterial disease detection, awareness, and treatment in primary care. JAMA 2001; 286: 1317-1324

8 Tunstall-Pedoe H, Peters SAE, Woodward M, et al. Twenty-year predictors of peripheral arterial disease compared with coronary heart disease in the Scottish Heart Health Extended Cohort (SHHEC). J Am Heart Assoc 2017; 6 : e005967.

9 Lin MS, Hsu KY, Chen YJ, et al. Prevalence and risk factors of asymptomatic peripheral arterial disease in patients with COPD in Taiwan. PLoS One 2013; 8: e64714.

10 Pecci R, Aguado JD, Rivo ABS, et al. Peripheral arterial disease in patients with chronic obstructive pulmonary disease. Int Angiol 2012; 31: 444-453.

11 Houben-Wilke S, Jorres RA, Bals R, et al. Peripheral artery disease and its clinical relevance in patients with chronic obstructive pulmonary disease in the COPD and Systemic Consequences-Comorbidities Network Study. Am J Respir Crit Care Med 2017; 195: 189-197.

12 Morgan AD, Rothnie KJ, Bhaskaran K, et al. Chronic obstructive pulmonary disease and the risk of 12 cardiovascular diseases: a population-based study using UK primary care data. Thorax 2018; 73: 877-879.

13 Ikram MA, Brusselle GGO, Murad SD, et al. The Rotterdam Study: 2018 update on objectives, design and main results. Eur J Epidemiol 2017; 32: 807-850.

14 Terzikhan N, Verhamme KM, Hofman A, et al. Prevalence and incidence of COPD in smokers and non-smokers: the Rotterdam Study. Eur J Epidemiol 2016; 31: 785-792.

15 Meijer WT, Hoes AW, Rutgers D, et al. Peripheral arterial disease in the elderly: the Rotterdam Study. Arterioscler Thromb Vasc Biol 1998; 18: 185-192.

16 Rose GA, Blackburn H, Gillum RF, et al. Cardiovascular survey methods. Geneva, World Health Organization, 1982.

17 Leening MJ, Kavousi M, Heeringa J, et al. Methods of data collection and definitions of cardiac outcomes in the Rotterdam Study. Eur J Epidemiol 2012; 27: 173-185.

18 Hofman A, Brusselle GG, Darwish Murad S, et al. The Rotterdam Study: 2016 objectives and design update. Eur J Epidemiol 2015; 30: 661-708.

19 Braun KVE, Dhana K, de Vries PS, et al. Epigenome-wide association study (EWAS) on lipids: the Rotterdam Study. Clin Epigenetics 2017; 9: 15.

20 Broekhuizen R, Wouters EF, Creutzberg EC, et al. Raised CRP levels mark metabolic and functional impairment in advanced COPD. Thorax 2006; 61: 17-22.

21 Diehm C, Allenberg JR, Pittrow D, et al. Mortality and vascular morbidity in older adults with asymptomatic versus symptomatic peripheral artery disease. Circulation 2009; 120: 2053-2061.

22 Alahdab F, Wang AT, Elraiyah TA, et al. A systematic review for the screening for peripheral arterial disease in asymptomatic patients. J Vasc Surg 2015; 61: 42S-53S.

23 Vaidya A, Joore MA, Ten Cate-Hoek AJ, et al. Screen or not to screen for peripheral arterial disease: guidance from a decision model. BMC Public Health 2014; 14: 89. 